\title{
Models of coupled population oscillators using 1-D maps
}

\author{
John Vandermeer, Andrew Kaufmann \\ Department of Biology, University of Michigan, 830 N. University, Ann Arbor, \\ MI 48109-1048, USA \\ e-mail: jvander@umich.edu
}

\begin{abstract}
Coupled population oscillators are investigated with the use of coupled logistic maps. Two forms of coupling are employed, reproductive and density. Three biologically distinct situations are investigated: populations independently oscillating in a two point cycle, populations independently chaotic, and populations independently approach a stable point. Both entrained and phase reversed patterns are observed along with complicated forms of chaos as the coupling parameters are varied.
\end{abstract}

Key words: 1 -D maps - Oscillators - Populations - Chaos

\section{Introduction}

Coupled oscillators have been central to developments in non-linear dynamics (Abrahams and Shaw, 1988; Arrowsmith and Place, 1990; Jackson, 1991), from the classic problem of coupled electronic oscillators (van der Pol and van der Mark, 1927) to biological circadian rythms (Winfree, 1967). In phenomena as diverse as simple coupled pendula and neural signals in slime molds (Sachsenmaier, Remy and Plattner-Schobel, 1972) complicated chaotic patterns can result from linking oscillators. While biological populations frequently illustrate oscillatory tendencies (Huffaker, 1958; May, 1981; Naeem, personal communication), only recently has the behavior of population models been examined from the point of view of coupled oscillators (Vandermeer, 1993, 1994, 1996), although other biological oscillations have been so examined, ranging from cardiac cells (Torre, 1976) to firefly flashes (Buck and Buck, 1976).

Modeling fluctuating populations with differential equations is a standard technique in ecology, but such an approach is frequently augmented by the use of $1 \mathrm{D}$ maps since the latter frequently lay bare the qualitative nature of the underlying biological dynamics more easily or at least more heuristically than continuous models. Furthermore, many biological systems are inherently 
discrete and more appropriately modeled with discrete systems. Thus 1D map forms of the classic exponential and logistic differential equation are now commonly found in ecology texts and the Nicolson-Baily version of the relationship between predator and prey is routinely used in place of the Lotka Volterra equations in many applications (e.g., Hassel et al., 1991).

Following this tradition, it seems appropriate to explore the behavior of coupled oscillators when the parent oscillator is a simple 1D population model. As a rough model of a two dimensional predator-prey oscillator, the humped 1D map has already been used (May, 1977), and it does not take a great deal of qualitative argument to see why (If predator is large this year, the prey will be rarer next year; If prey is abundant this year, predator will be more abundant next year; a very large population of either predator or prey probably results in a smaller population thereof next year, while a small population of either probably becomes larger next year, etc. . .). Using the 1D approach we can either look at prey dynamics or predator dynamics through time, presuming first that the dynamics of predator and prey together drive the emergent dynamics of either population examined separately, and second that we can describe those emergent dynamics approximately with a 1D map.

In particular we here investigate the behavior of coupling two 1D logistic models together, exploring the resulting patterns of phase locking, chaos generation, and chaos abatement. Such results are of interest not only to the simple problem of two populations linked together, but possibly provide insights into the spatial distribution of populations, as suggested in the discussion. There is already a significant literature on this topic, much of which we refer to in the course of the following discussion, but a systematic treatment has yet to appear (Jackson, 1991: p. 434).

We shall be concerned with the simple logistic 1D map,

$$
x_{t+1}=r x_{t}\left(1-x_{t}\right)
$$

where $x$ is the biomass of population 1, and $r$ the rate of population increase. The dynamics of this map are very well known (e.g. Devany, 1986: Feigenbaum, 1979; May, 1976), and will not be discussed.

\section{Phase locking in the uncoupled system}

We begin by defining a second population as having the same dynamics,

$$
y_{t+1}=r y_{t}\left(1-y_{t}\right)
$$

where $y$ is the biomass of population 2 and $r$ is as before. In this study the value of $r$ remains constant from population to population, that is, the system is always symmetrical.

With the proper choice of $r(3<r<3.449)$ there is a permanent two point cycle which we take to be a model of a permanently cycling system, either because of a predator/prey relationship, or for any other reason. Restricting our analysis in this section to only those maps exhibiting stable 2 point cycles, 

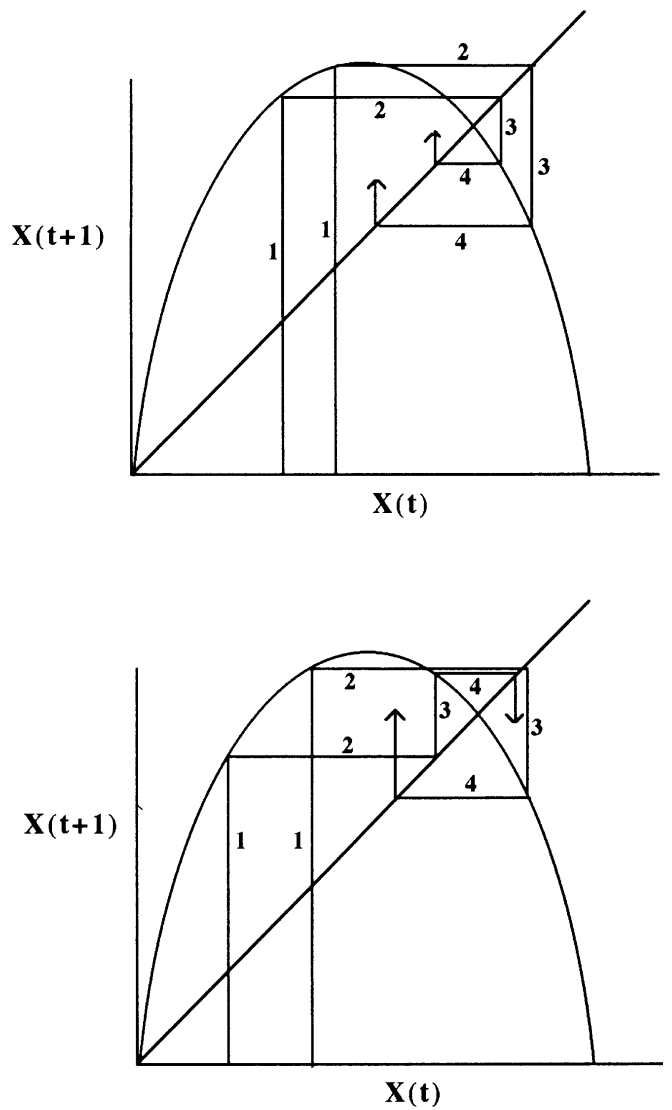

Fig. 1. Illustration of the mechanism of entrainment (top graph) versus phase reversal (bottom graph), in the simple logistic map

we make the obvious observation that two independent (uncoupled) populations exhibiting this behavior must be either perfectly in phase or perfectly out of phase, the initiating values determining whether the phase locking is in or out of phase. In Fig. 1 we illustrate the obvious criterion for whether trajectories will be in or out of phase, Fig. 1a illustrating two trajectories that will remain entrained (in phase) and Fig. 1b illustrating two trajectories that will reverse their oscillations to become phase reversed (we shall follow the convention henceforth of refering to populations in phase as "entrained" and populations out of phase as "phase reversed").

We define a set of points $A$ on the interval $x=0,1$, such that any two trajectories which are initiated in $A$ will eventually become entrained, what we call the "uniform trajectory set." Let $a_{1}$ be the equilibrium point (the value of $x$ that satisfies $x=\operatorname{rx}(1-x)$, namely $\left.a_{1}=(r-1) / r\right)$. Let $a_{1}^{\prime}=f^{-1}\left(a_{1}\right)$. Since we are dealing specifically with the logistic map, we can define the inverse function as the two roots of the equation considering $x_{t+1}$ as an input variable, or

$$
x_{t}=g\left(x_{t+1}\right)=0.5 \pm\left[0.25-x_{t+1} / r\right]^{0.5} .
$$


Substituting $a_{1}$ for the input variable, we thus compute,

$$
a_{n}, a_{\mathrm{n}}^{\prime}=g\left(a_{1}\right)^{n}
$$

where the superscript indicates iterations rather than exponentiation. Then the uniform trajectory set is delimited by

$$
x e\left\{a_{n}, a_{n}^{\prime}\right\}
$$

where $n$ is either always even or always odd. Thus there are two uniform trajectory sets, one defined by $a_{1}, a_{1}^{\prime} ; a_{3}, a_{3}^{\prime}, a_{5}, a_{5}^{\prime} ; \ldots$ the other by $a_{2}, a_{2}^{\prime}$; $a_{4}, a_{4}^{\prime}, \ldots$. Plotting these two sets on both $x$ and $y$ axis we can thus analytically plot the uniform trajectory sets for the two populations taken together, even though they are completely independent of one another. Such a plot is pictured in Fig. 2.

The delimited areas in Fig. 2 represent basins of attraction - any two trajectories initiated in the shaded area (i.e. in the same uniform trajectory set) will entrain, whereas any two trajectories initiated in an unshaded area (i.e. opposite uniform trajectory sets) will come to be phase reversed. These basins give a complete picture of the relative phase behavior of the uncoupled system and provide a springboard for studying the changes induced by coupling.
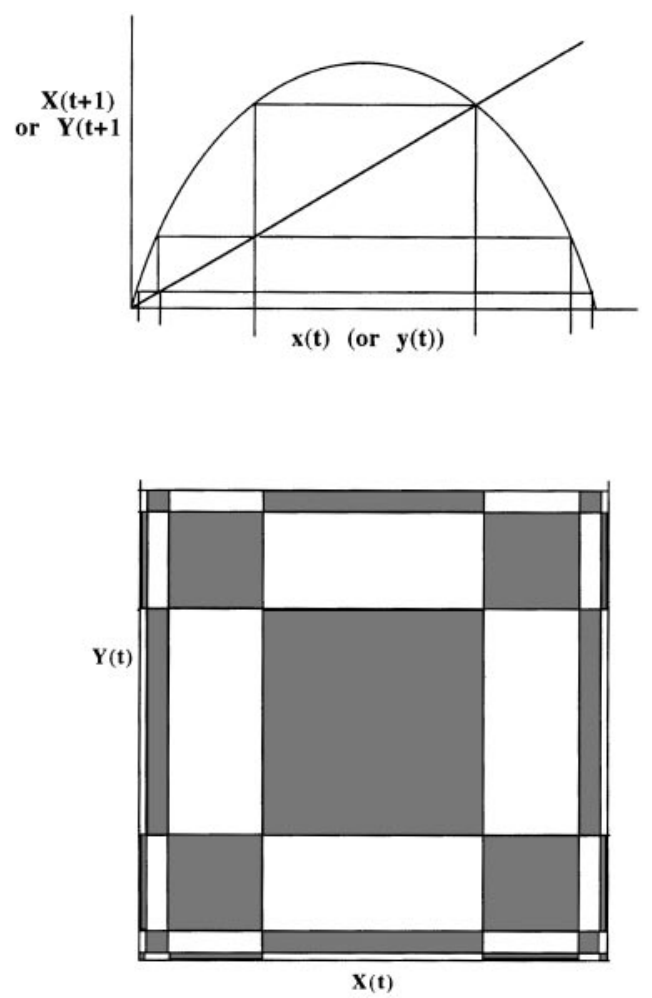

Fig. 2. Construction of phase reversed and entrainment basins based on the simple logistic map. Projections in top graph define limits on the various basins illustrated in bottom graph. Shaded areas are entrainment basins, unshaded areas are phase reversal basins 


\section{Entrainment and reversal basins in the coupled system}

If $x$ and $y$ are the state variables of two populations, both of which are oscillating identically, but not necessarily either in or out of phase, we now wish to weakly couple them and study their joint behavior. Biologically this implies that if the two populations are predators oscillating in reponse to two other unseen prey species, we allow the predators to occasionally eat the alternate prey. Or if they are simply two identical populations but islolated from one another (fish populations in neighboring lakes, insect populations on neighboring mountains, etc...), coupling might imply a small amount of migration between the populations (the lakes connected by a swamp that allows some small interchange between the fish populations, or a corridor of appropriate vegetation connecting two mountain top insect populations). The two populations together form a "meta population."

When dealing with such idealized populations as those represented by the logistic map there are a limited number of qualitatively distinct ways in which the populations may become coupled. We distinguish between the case in which individuals switch populations for the purpose of reproduction only (reproductive coupling) from the case in which the migrant individuals affect the density response of the receiving population but do not contribute to the reproductive effort of their adopted homeland (density coupling).

Allowing a fraction $b$ of the populations to exchange each time unit, we can write,

$$
\begin{aligned}
& x_{t+1}=r\left[x_{t}(1-b)+b y_{t}\right]\left(1-x_{t}\right) \\
& y_{t+1}=r\left[y_{t}(1-b)+b x_{t}\right]\left(1-y_{t}\right)
\end{aligned}
$$

as the model for reproductive coupling, and

$$
\begin{aligned}
& x_{t+1}=r x_{t}\left(1-x_{t}(1-b)-b y_{t}\right) \\
& y_{t+1}=r y_{t}\left(1-y_{t}(1-b)+b x_{t}\right)
\end{aligned}
$$

as the model for density coupling.

We consider first reproductive coupling, Eqs. (2a) and (b). Equation (2a) can be rewritten as,

$$
x_{t+1}=r x_{t}\left(1-x_{t}\right)-r b\left(x_{t}-y_{t}\right)\left(1-x_{t}\right),
$$

which makes clear that it consists of two parts, the first term being the original map and the second term being that which is subtracted from the original model to form the "effective" return map for the variable $x$. We can thus explore the qualitative behavior of Eq. (4) as if it were a deviation from the original logistic map.

First note that if the population is in a perfectly entrained state, $y_{t}=x_{t}$ and Eq. (4) reverts to the original logistic map. Thus the entrained state (if one exists in the $2 \mathrm{D}$ model) will have a two point cycle that is identical to the original map. 
Second, note that if the population is phase reversed, $y_{t} \neq x_{t}$, the new apparent map will be either above or below the original logistic map, depending on the value of $y$. In Fig. 3 we illustrate both the original logistic map and Eq. (4), for arbitrary values of $y$, under the assumption that high $x$ implies low $y$ and vice versa, the basic idea behind phase reversal. As illustrated in Fig. 3, the basin of attraction must become distorted, the first main consequence of coupling the equations.

We note that empirically it is clear that reproductive coupling leads to the gradual decomposition of the entrainment cycle, as illustrated by the series of basin maps in Fig. 4. Note the gradual disappearance of the entrainment basin (except for the principal diagonal), and the slight deformity along the principal diagonal axis.

If we now consider density coupling, we have,

$$
x_{t+1}=r x_{t}\left(1-x_{t}\right)+r b x_{t}\left(x_{t}-y_{t}\right),
$$

which also can be interpreted as the original logistic plus a modifier, but this time the modifier is added to the original map. As before, the addition will be zero when the system is in phase, and either greater than zero (for large $x$ ) or less than zero (for small $x$ ) when the system is out of phase. Following the same logic applied to the case of reproductive coupling, we similarly conclude that as coupling becomes more intense, the basins themselves should become deformed. In Fig. 5 we illustrate the series of basins deforming until the phase reversed basin completely disappears.

It is thus possible to conclude qualitatively that the basins become deformed, and that reproductive coupling eventually leads to complete phase reversal while density coupling eventually leads to complete entrainment.

We can determine the precise point of loss of either the entrainment or reversal basins by analyzing the doubly iterated map, namely, for reproductive coupling,

$$
\begin{aligned}
x_{t+2}= & r^{2}\left[\left\{x_{t}(1-b)+b y_{t}\right\}\left(1-x_{t}\right)(1-b)+b\left\{y_{t}(1-b)+b y_{t}\right\}\left(1-y_{t}\right)\right] \\
& \times\left[\left(1-r\left\{x_{t}(1-b)+b y_{t}\right\}\left(1-x_{t}\right)\right)\right]
\end{aligned}
$$

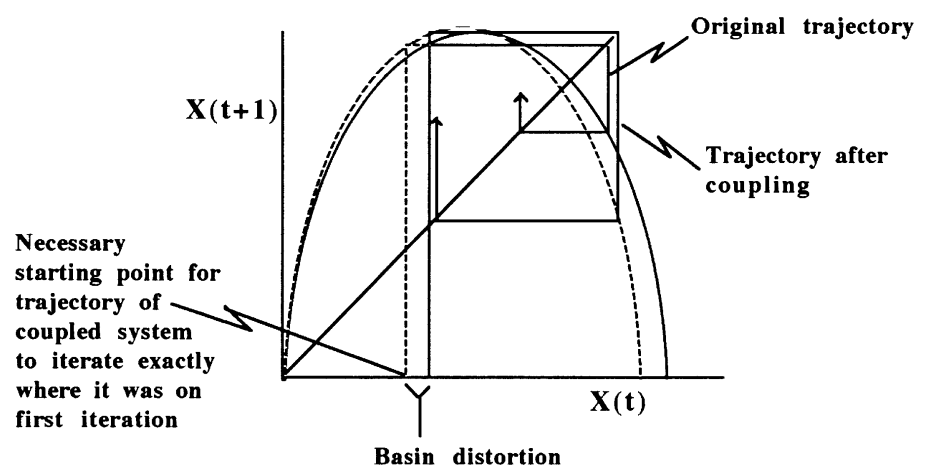

Fig. 3. Qualitative depiction of basin distortion due to slight shift in the shape of the logistic map, as dictated by Eq. (4) 

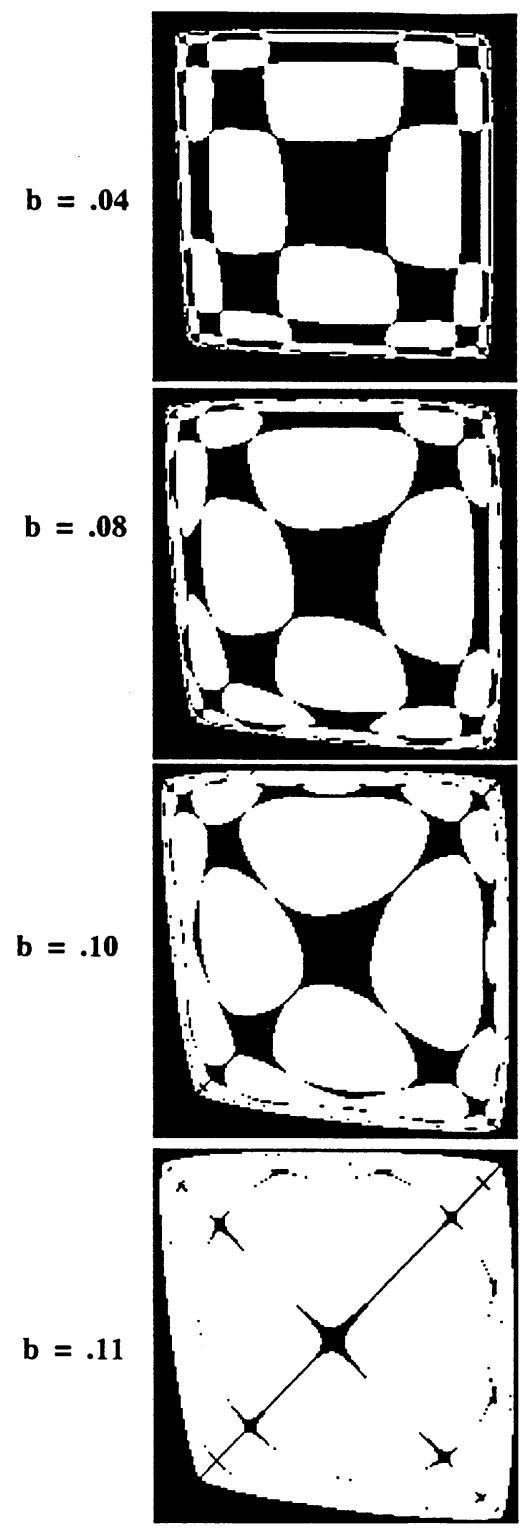

Fig. 4. Deformation of basins due to reproductive coupling. Shaded areas represent entrainment basins and clear areas phase reversal basins. As coupling increases, the entrainment basins tend to disappear

and setting $x_{t+2}=x_{t}$. Performing the same operation on the other population we can plot Eq. (6) in $x, y$ space and examine the position of the roots at various values of $b$. By inspection it is obvious that Eq. (6) has eight roots, four of which correspond to the four equilibrium points in the coupled system (two points for the entrained cycle and two points for the phase reversed cycle). In Fig. 6 we plot all real positive values for Eq. (6) (along with the equivalent equation for $y$ ) for three values of $b$, illustrating how the intersection of these 

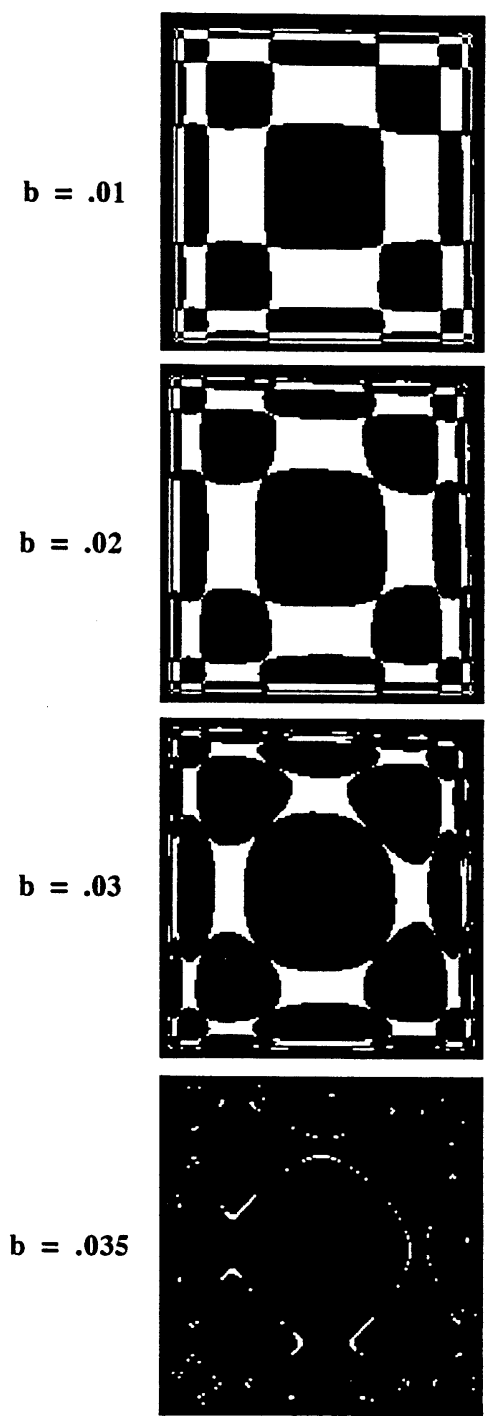

Fig. 5. Deformation of basins due to density coupling. Shaded areas represent entrainment basins and clear areas phase reversal basins. As coupling increases, the phase reversal basins tend to disappear

equations stipulates both the phase reversed and entrained cycle, and how the entrainment points disappear as $b$ approachs 15 .

If the coupling is managed through the density connection, we obtain Eqs. (3a) and (b), the first of which can be rewritten as,

$$
x_{t+1}=r x_{t}\left(1-x_{t}\right)+r x_{t} b\left(x_{t}-y_{t}\right)
$$

The doubly iterated map is simply,

$$
\begin{aligned}
x_{t+2}= & r^{2} x_{t}\left(1-x_{t}\right)+r x_{t} b\left(x_{t}-y_{t}\right)\left(1-r x_{t}\left(1-x_{t}\right)+r x_{t} b\left(x_{t}-y_{t}\right)\right) \\
& +r\left[r x_{t}\left(1-x_{t}\right)+r x_{t} b\left(x_{t}-y_{t}\right)\right] b\left(r x_{t}\left(1-x_{t}\right)+r x_{t} b\left(x_{t}-y_{t}\right)-y_{t}\right) .
\end{aligned}
$$



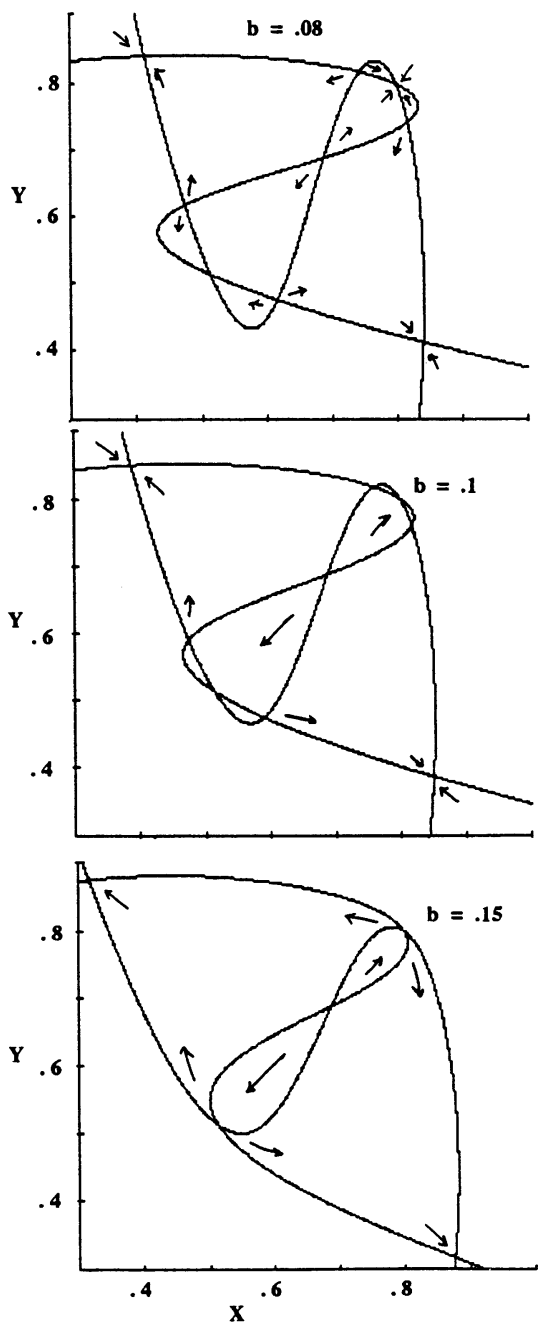

Fig. 6. Graph of Eq. (6) illustrating the changes in intersection points (and loss of entrainment cycle) following changes in coupling values

As before, the isoclines for density coupling are presented in Fig. 7 for various values of $b$, illustrating the loss of the reversed cycle at approximately $b=0.035$.

\section{The initial Hopf bifurcation and its deformation}

Considering the reproductive coupling form only, as coupling strength increases both points undergo a Hopf bifurcation at approximately $b=0.1181$ (in all of this we retain the value of $r=3.2$ as representative of the 1D system being in a two point cycle). The behavior for values of $b$ slightly greater than the Hopf bifurcation value can be most easily studied with the doubly iterated 


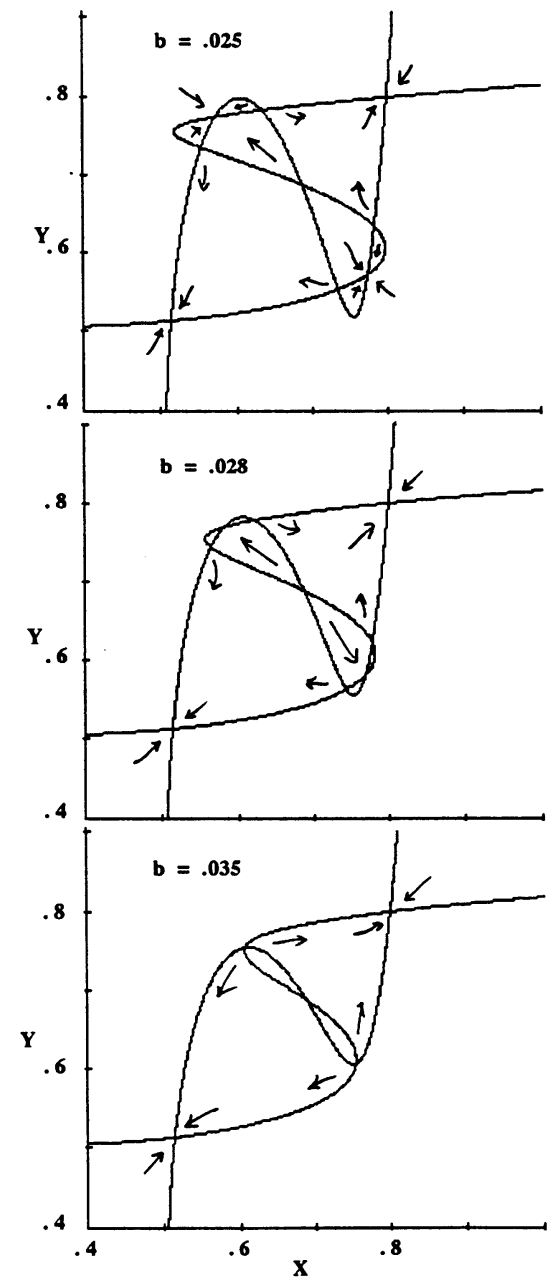

Fig. 7. Graph of Eq. (7) illustrating the changes in intersection points (and loss of phase reversed cycle) following changes in coupling values

map (Eq. (6)), in which case each successive point on the attractor appears in a regular sequence, as illustrated in Fig. 8 for two representative values of $b$. This sequence is reminiscent of the pattern obtained by the simple circle/sin map (Cvitanovic et al., 1990; Bohr et al., 1984), and thus suggests an appropriate analytical framework for its study.

Letting $y^{*}$ and $x^{*}$ represent the mean values of $x$ and $y$ obtained from iterating Eq. (6), we convert to polar coordinates arround the point $\left\{x^{*}, y^{*}\right\}$,

$$
\begin{aligned}
& \rho=\left[\left(x-x^{*}\right)^{2}+\left(y-y^{*}\right)^{2}\right]^{1 / 2} \\
& \theta=\sin ^{-1}\left[\left(x-x^{*}\right) / \rho\right]
\end{aligned}
$$

For $b=0.134$, we plot $\theta(t+1)$ against $\theta(t)$ after eliminating transients, as illustrated in Fig. 9. The outlines of the standard circle map can be easily discerned in Fig. 9. 

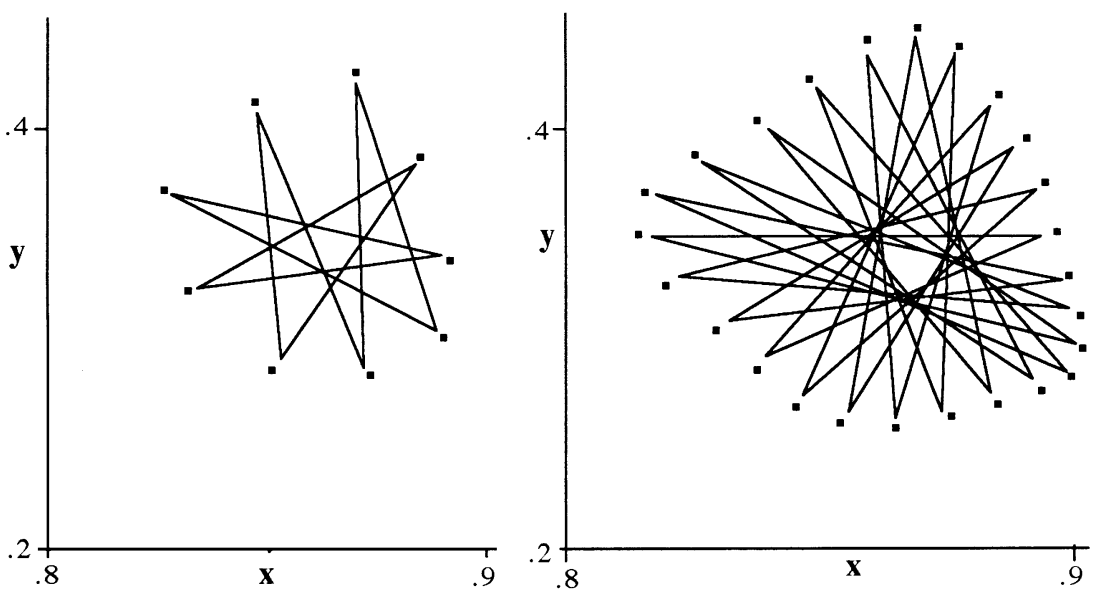

Fig. 8. Sequence of points visited in a 9 point cycle resulting from a coupling value of 0.134 (left graph) and a 24 point cycle resulting from a coupling value of 0.142165 . Lines connecting the points illustrate the sequence of visitation of points every other time interval (since these are representations of only half of the qualitatively phase-reversed cycle). The sequence in both cases is qualitatively consistent with expectations from a circle map

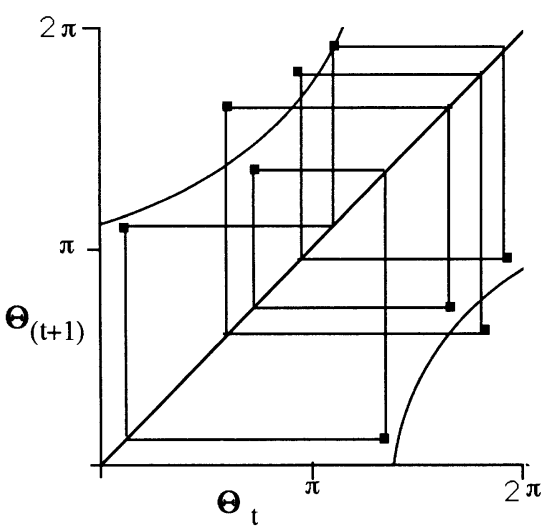

Fig. 9. Approximate circle map for reproductively coupled logistics with a 9 point cycle (see Fig. 8)

Using polar coordinates we illustrate the transformation of the Hopfbifurcated attractor as the connection of the two systems is increased. In Fig. 10 we show the bifurcation diagram for $\theta$ as the coupling parameter increases. Note the period doubling-like behavior, interspersed with quasiperiodic trajectories.

Referring to Fig. 10, the point at which the torus-like behavior clearly breaks down is about $c=0.157$. We illustrate one side of the attractor for this value of $c$ in Fig. 11. Note that at this point the attractor remains formally a torus, with the points seemingly dense. Thus, as we move from $c=0.134$ (at the Hopf bifurcation) to $c=0.157$, the original torus of the Hopf bifurcation undergoes various transformations, all of which appear to be topologically 


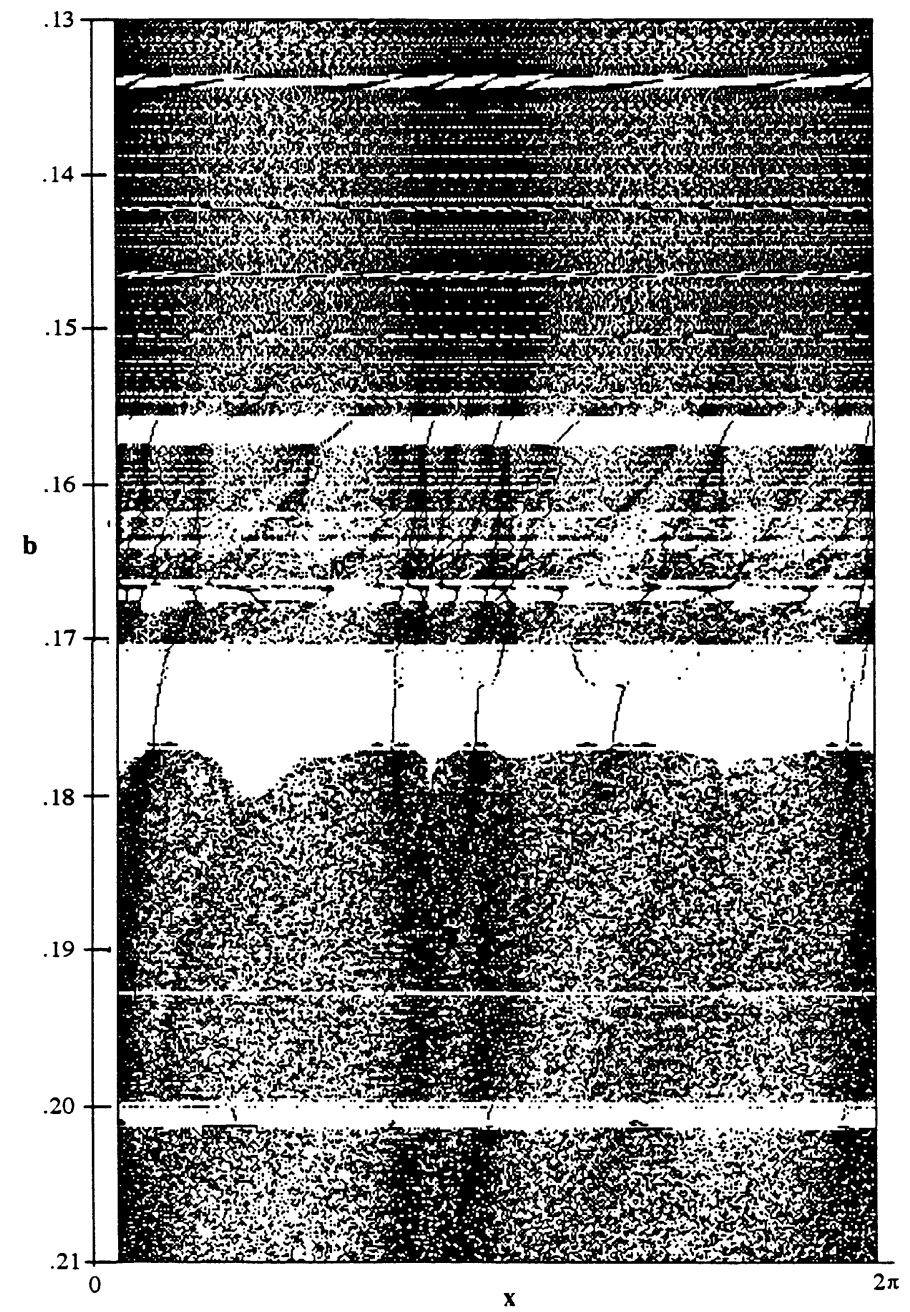

Fig. 10. Bifurcation diagram of the transformed variable ( $x$ refers to the angular polar coordinate) illustrating a complicated range of behaviors for the system. The 9 point cycle illustrated in Fig. 8 is clearly visible at the 0.134 parameter value, as is the initiation of breakup of torroidal behavior near 0.157 , followed by complicated changes in strange attractors and an apparently infinite number of periodic windows both before and after the breakup

homeomorphic. In Fig. 12 we illustrate how these changes procede beyond $c=0.157$ with graphs of $\theta$ versus $\rho$. It is clear that the mean and variance in $\rho$ over this particular region offers a quantitative estimate of the deformation of the original torus that emerged at the time of the Hopf bifurcation. This pattern is quite similar to the detailed analysis of Kaneko (1983) who summarizes changes in the torus in four steps: (i) the torus appears through a Hopf bifurcation; (ii) the torus becomes distorted (as can be seen in the 


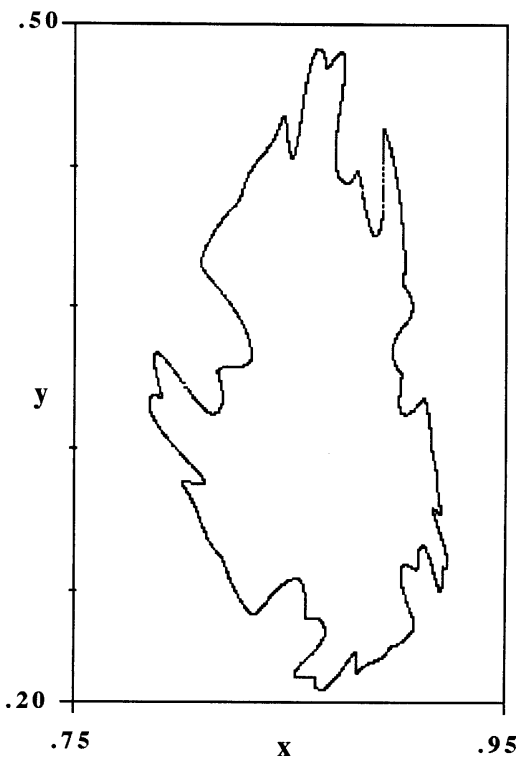

Fig. 11. Details of one half of the attractor (reproductive coupling) at $b=0.161$, during the breakup of toroidal behavior
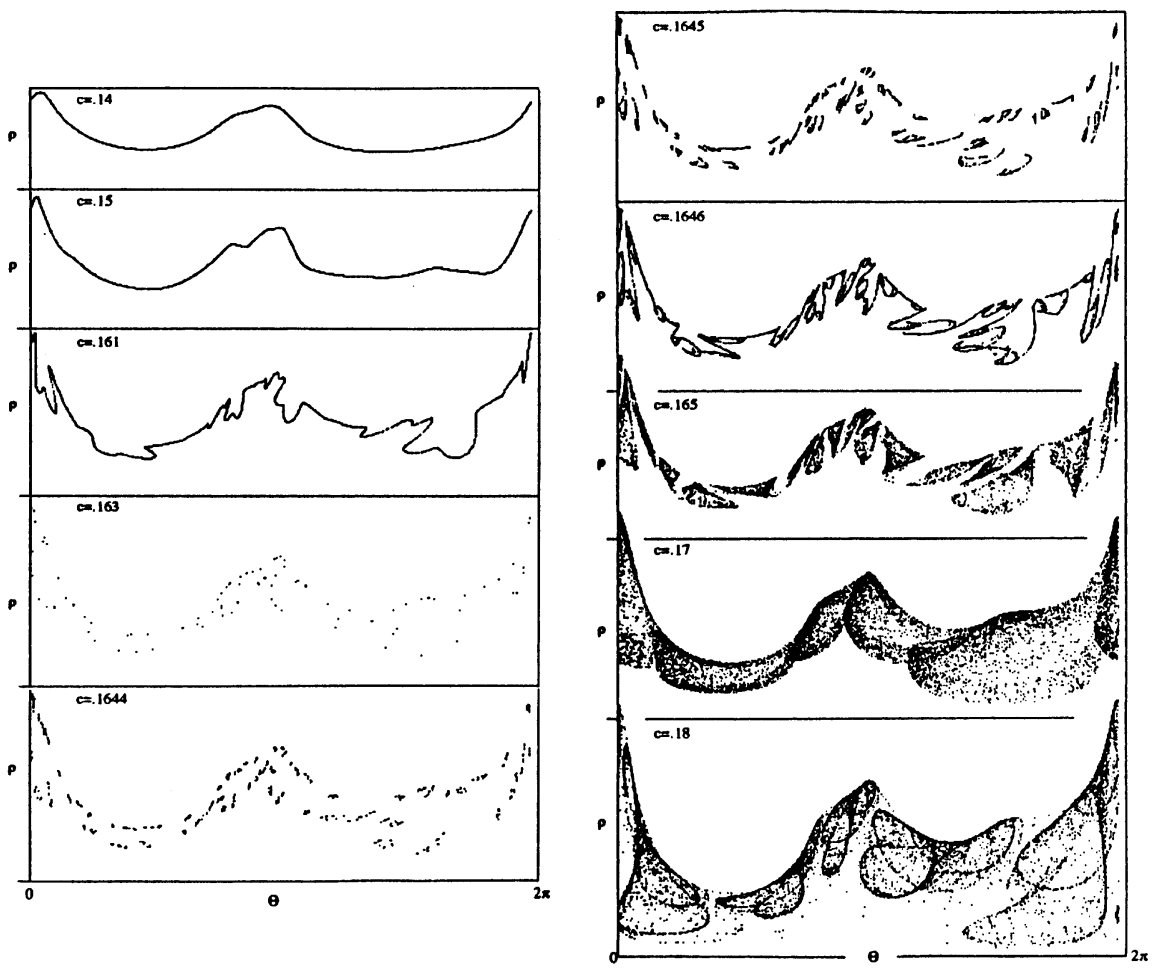

Fig. 12. Changes in one half of the attractor, represented in polar coordinates, during breakup of torroidal behavior, illustrating most of the qualitatively distinct forms of attractors (compare to the bifurcation diagram in Fig. 11) 


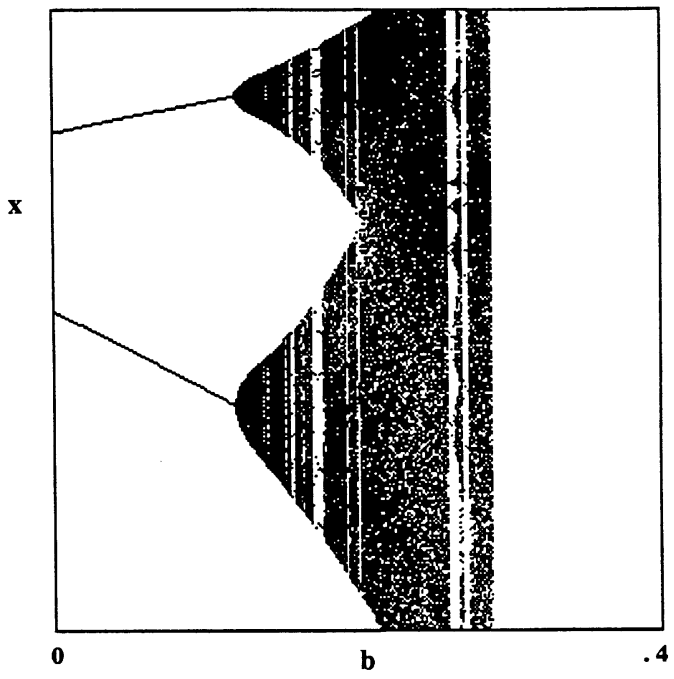

Fig. 13. Bifurcation diagram for the coupling parameter $b$ in the case of $r=3.2$ for reproductive coupling

various graphs in Fig. 12); (iii) regions of frequency lockings increase (forming a devil's staircase); and (iv) chaos appears through a period-doubling or tangent bifurcation of some frequency-locked cycle at some value of the bifurcation parameter. Yuan et al. (1983) made similar observations, and suggested that the deformations of the torus (e.g. Figs. 11 and 12) was evidence that the Ruelle-Takens-Newhouse scenario of chaos formation was operative.

The deformation of what had been the original Hopf bifurcation continues in a complicated fashion as the coupling strength continues as shown in Fig. 12. The bifurcation diagram is illustrated in Fig. 13 and a series of attractors shown in Fig. 14. Note how the two sections of the attractors, corresponding to the original circular arrangement that arose from the Hopf bifurcation, enlarge, become more complicated, and eventually merge to a large and very complicated attractor, as illustrated in Fig. 14.

Thus, the overall pattern as reproductive coupling strength increases, is phase locking in either an entrained or reversed form, followed by the loss of the entrainment cycle, the phase reverse situation remaining. Subsequently the two fixed points of the phase reversed cycle undergo a Hopf bifurcation followed by a series of complex deformations, eventually resulting in complex strange attractors.

The interesting and complex behavior of the system under reproductive coupling is not repeated in the case of density coupling (Eq. (3)), in which the loss of the reversal basins simply results in entrainment, and furthur coupling leads to no other qualitatively distinct behavior.

\section{Coupling chaotic systems}

The question naturally arises as to what pattern will be formed if the parent equations are themselves chaotic, a point to which we now turn. Using the 


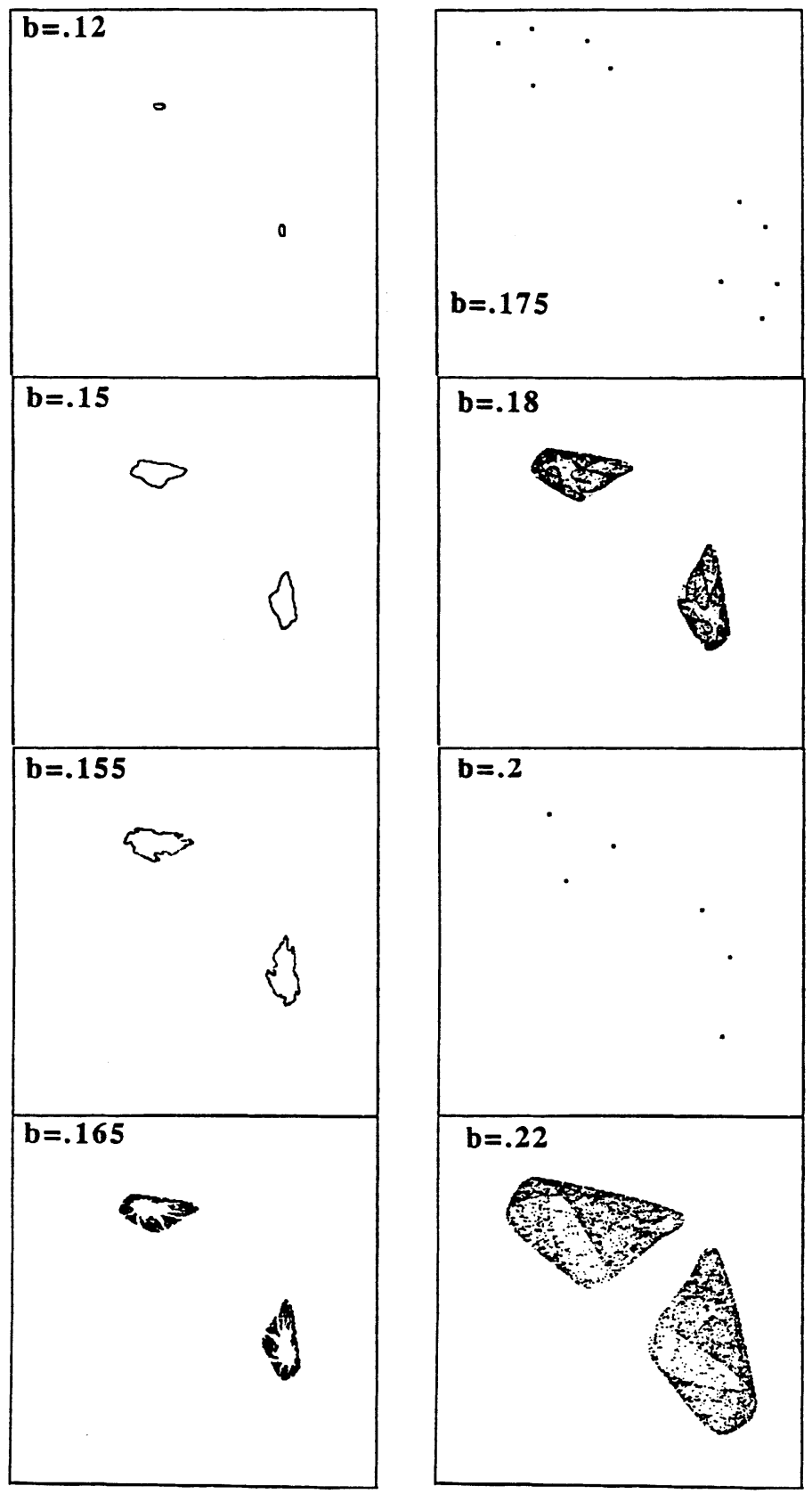

Fig. 14. A sampling of phase reversed attractors resulting from increasing coupling strength in the reproductively coupled system with $r=3.2$ 
running example of $r=3.6$, it is relatively simple to delimit the boundaries of the attractor that represents the two unconnected systems as a lower limit of 4- $r$ and and upper limit of $r / 4$. Thus the attractor in $x, y$ space will be a moreor-less random distribution of points within the square defined by $y=4-r$, $r / 4$ and $x=4-r, r / 4$.

Reproductive coupling of these equations in the chaotic zone produces a splitting of the initial attractor, such that chaotic analogues of entrainment and phase reversal are created. Thus, loosely coupling the two equations produces two distinct and alternative attractors, one of which is qualitatively entrainment the other phase reversal, both of which are presented in Fig. 15. As with entrained or phased-reversed stable oscillations, one can compute basins of attraction, which, in this case appear qualitatively identical to the basins obtained for the stable points (see Figs. 4 and 5).

Increasing the degree of coupling causes the entrainment attractor to further split, such that four zones are systematically visited, but within each zone the system remains chaotic (Figs. 16a and b). On the other hand, the same increase in the coupling strength causes the phased reverse attractor to simply become larger. With further increases in coupling strength, the four sections of the entrainment attractor eventually emerge as four tori (Fig. 16c), still chaotic in the sense that no point on the attractor is ever repeated (in the present case we note only that no point is repeated after a very long run). Each of these tori appears qualitatively similar to the structures observed earlier following the Hopf bifurcation (see Figs. 11 and 12). These tori continue to become smaller and smaller, until they eventually undergo a reverse Hopf bifurcation and become single points, at which point the attractor is no longer

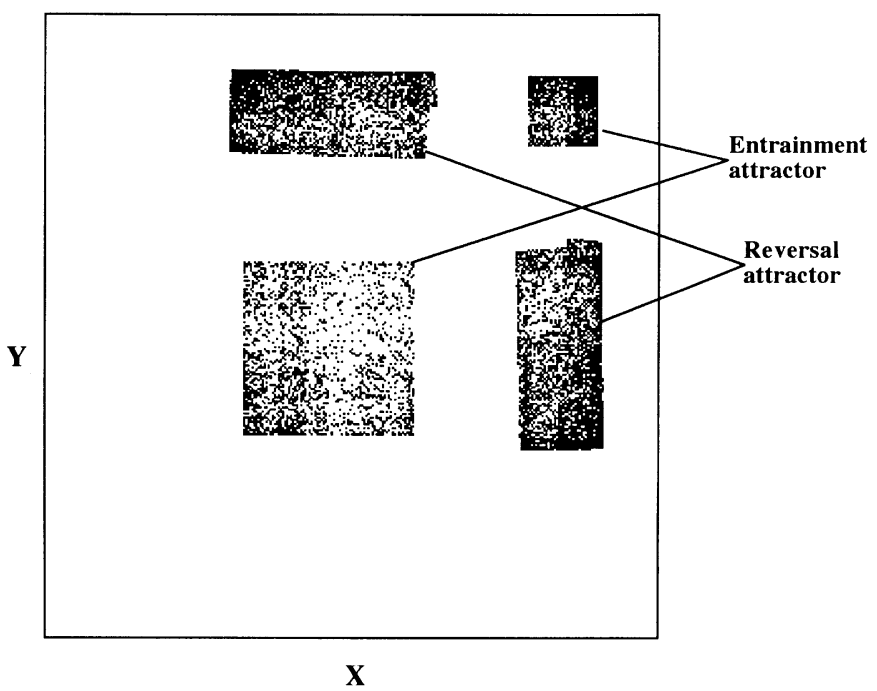

Fig. 15. The two alternative attractors in the very weakly coupled chaotic system (reproductive coupling parameter $=0.01, r=3.6$ ) 

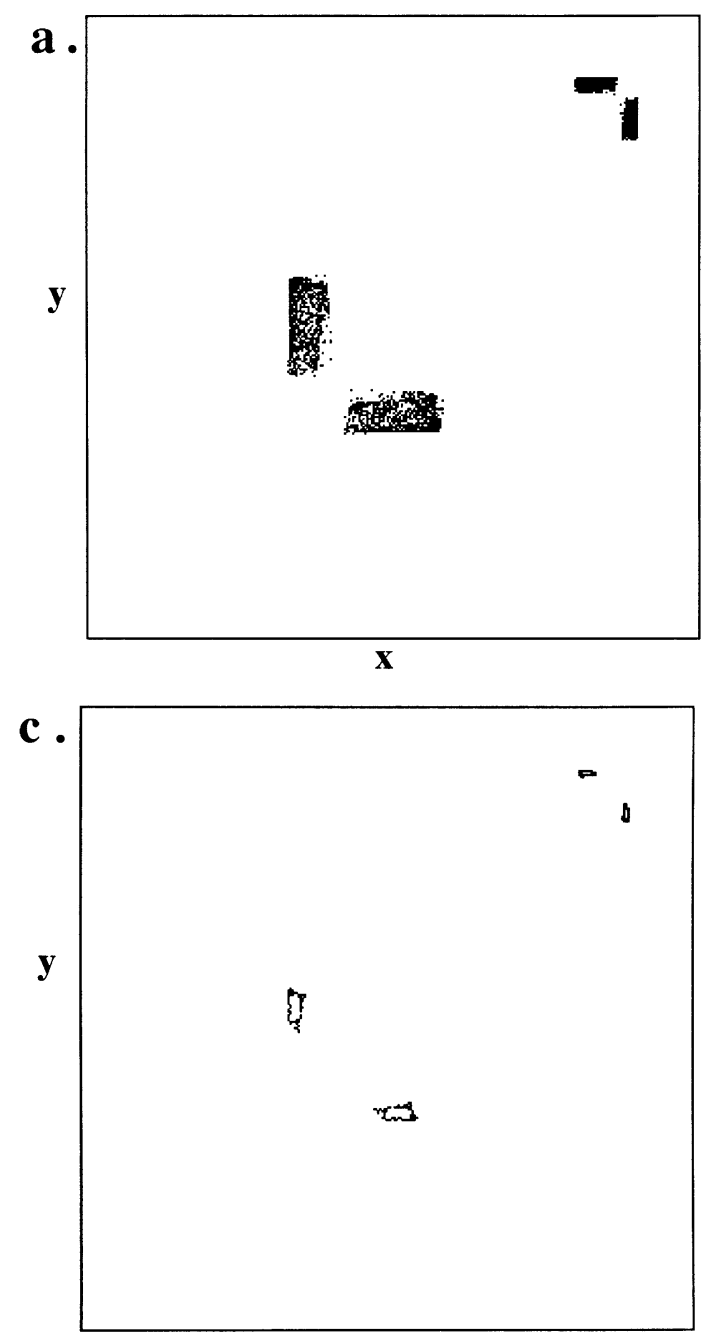

$\mathbf{x}$

Fig. 16a-d. Changes in the chaotic attractor resulting from coupling (reproductive). Parts $\mathbf{a}$ and $\mathbf{b}$ are similar to Fig. 15 with slightly larger couplings $(b=0.05)$. Part $\mathbf{c}$ illustrates the effective reconstruction of torroidal-like behavior from the originally chaotic system, through coupling $(b=0.1)$. Part $\mathbf{d}$ shows both attractors superimposed on the basins of attraction, just at the point of crisis (where a strange attractor intersects the basin of an alternative attractor)

chaotic, but represents a simple four point cycle, each point in the cycle the image of the former four part chaotic attractor shown in Fig. 16a.

Finally, with further increases in coupling, the area of the phase reversed attractor increases to the point that the basins of the entrainment attractor are intersected by the reversed attractor, the implications of which are, of course, 


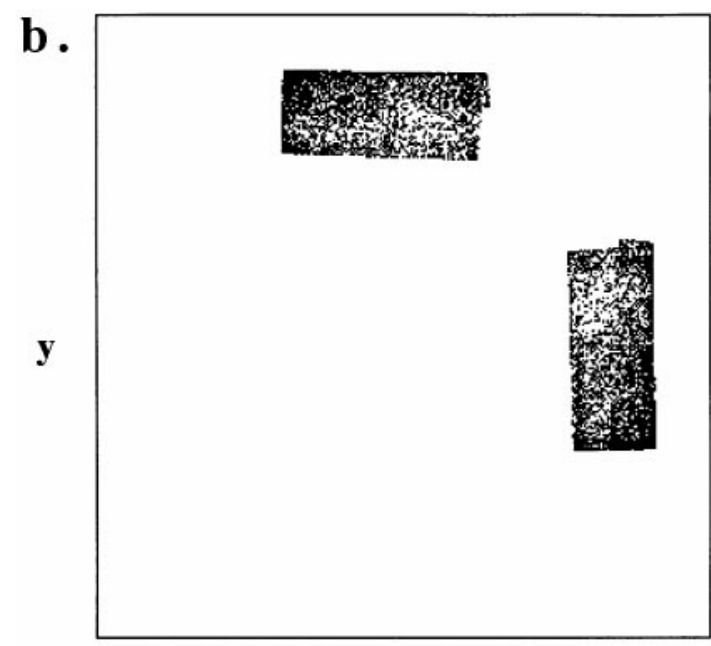

$\mathbf{x}$

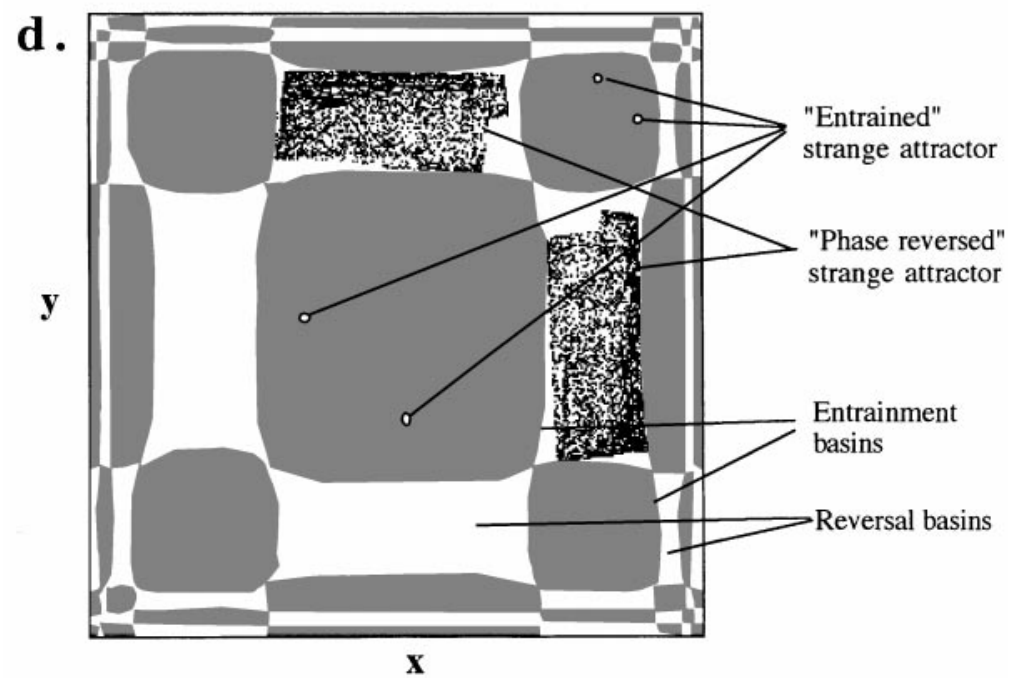

Fig. 16. Continued

that the phase reversed attractor is no longer a stable attractor. All trajectories within it will eventually escape into the basin of the four point attractor. This situation is illustrated for the case just before the reverse Hopf bifurcation in Fig. 16d. It is an interesting situation globally in that the eventual behavior of the system is a four point cycle, but, obviously, a chaotic attractor also exists. The latter is in principle impossible to actually see since it is unstable. It appears to be something analogous to a saddle point in that any system 
initiatied in a reversal basin will become established in this strange attractor, but since the attractor itself overlaps with the entrainment basins, any trajectory in the strange attractor will eventually become entrained in the four point entrainment cycle. Thus trajectories are first attracted to it yet later must diverge from it, making it like a saddle point, except that it is a chaotic attractor. This sort of behavior has been termed "crisis" and is discussed by Gu et al. (1984). We expect other examples of such "chaotic saddle attractors" will emerge in other contexts, and note the potential importance of a system that remains chaotic for potentially long periods of time, but eventually converges on a stable period.

All of the above behavior is conveniently summarized in bifurcation diagrams, both of which are shown in Fig. 17. Figure 17a shows the bifurcation diagram generated by initiating the system near its entrained state, while

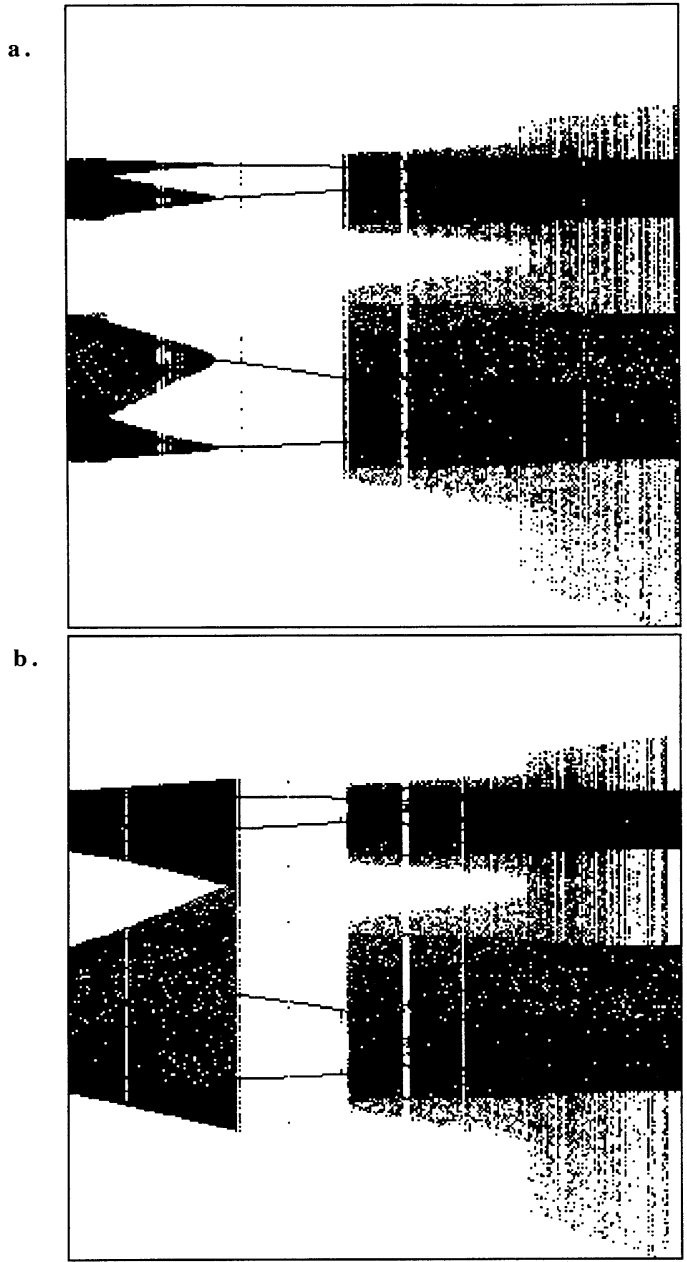

Fig. 17a, b. Bifurcation diagrams for $r=3.6$ (both parent logistics in the chaotic realm) beginning near the entrainment cycle (top graph) or near the phase-reversed cycle (bottom graph). Clearly, after the point of crisis both bifurcation diagrams are equal 

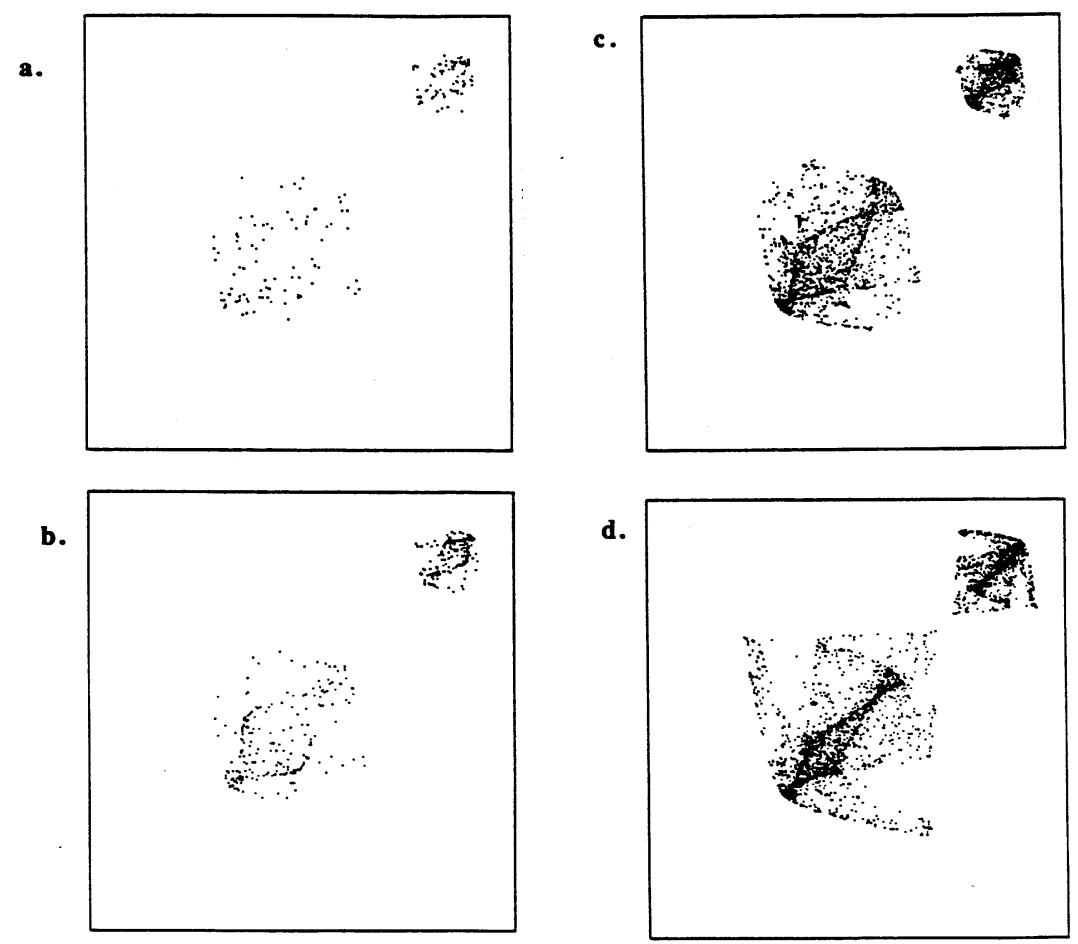

Fig. 18a-d. Examples of attractors with relatively high levels of coupling in the chaotic parent system $(r=3.6)$, $\mathbf{a}$ and $\mathbf{b}$ lower couplings $(b=0.05$ and 0.055$)$, $\mathbf{c}$ and $\mathbf{d}$ with relatively higher values $(b=0.07$ and 0.08$)$

Fig. 17b shows the bifurcation diagram generated by initiating the system near its phased reversed state. Most of the behavior patterns described above are easily seen in the left half of the bifurcation diagram, including the original switch from the attractor with just two areas to that of four through the reverse Hopf bifurcation to a four point cycle (see Fig. 17b). Also what may appear unusual behavior initially where the chaotic reversed phase attractor suddently converts to a four point cycle (see Fig. 17a), makes sense in the light of the type of bifurcation in which a chaotic attractor intersects the basin of attraction of a stable cycle (i.e. a crisis).

With further increases in coupling strength, the four point cycle bifurcates repeatedly into very high periodicity (Figs. 18a, b, and c), and eventually takes a peculiar shape symmetrical around the principal diagonal (Fig. 18d), and in two large patches more or less located in the position of the original patches (recall Fig. 16). Note that by the time coupling strength is 0.08 (Fig. 18d), there is a statistical concentration of all points near the principal diagonal.

Proceeding to yet further increases in coupling strength, a rather unusual behavior emerges, in which both populations are clearly chaotic, but track one another quite completely. To see this we simply form the measure $d=x t-y t$ 


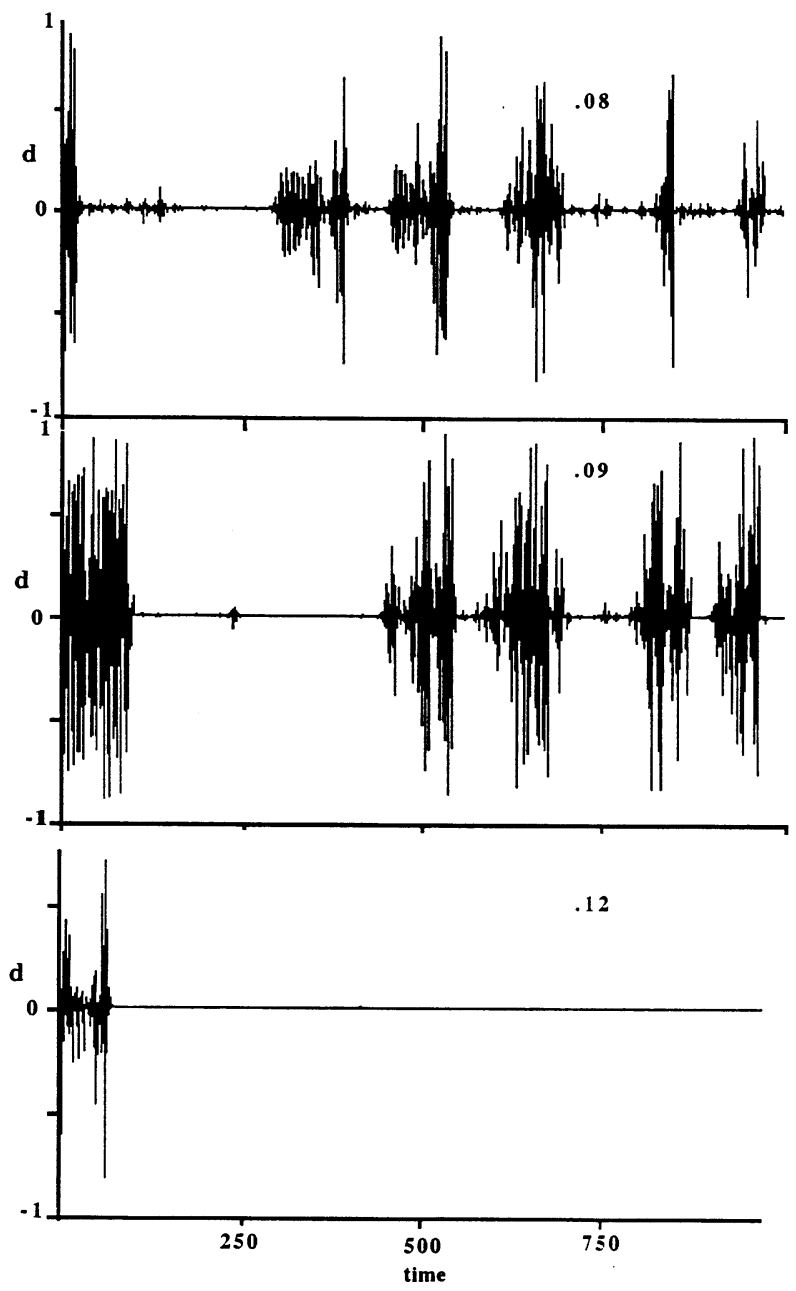

Fig. 19. The difference between the two systems, (population density of one minus population density of the other) over time. Note the tendency towards tracking especially evident when $b=0.09$, and completely synchronous with $b=0.12$

and plot $d$ over time. As shown in Fig. 19, for this range of coupling strength, $d$ tends towards zero, with bursts of deviations near to 1 and -1 , except when the coupling strength reaches a critical value, in which $d$ appears to be zero for all times. It should be emphasized that both populations are exhibiting chaotic behavior, nevertheless, their behavior is perfectly synchronized, a phenomenon previously noted in models of coupled lazers and dubbed "synchronized chaos" (Winful and Rahman, 1990). 


\section{Consequences of coupling non-oscillating populations}

While the purpose of this study in the first place was to mimic the behavior of two coupled oscillators, an interesting pattern emerges from the coupling of extremely stable populations. Setting the value of $r$ equal to 2.8, each parent population exhibits a stable one point cycle, well known in the literature (Lauwerier, 1986). Coupling such populations together would not a priori be expected to generate anything unusual. However, coupling the two populations at the level of $b=0.1$, a strange attractor is generated, as illustrated in Fig. 20. The complete bifurcation diagram for $r=2.8$ is illustrated in Fig. 21.

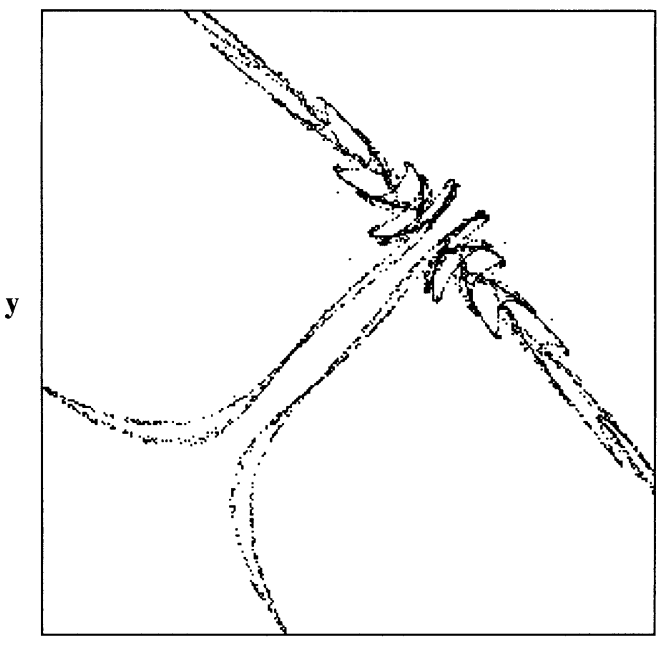

$\mathbf{x}$

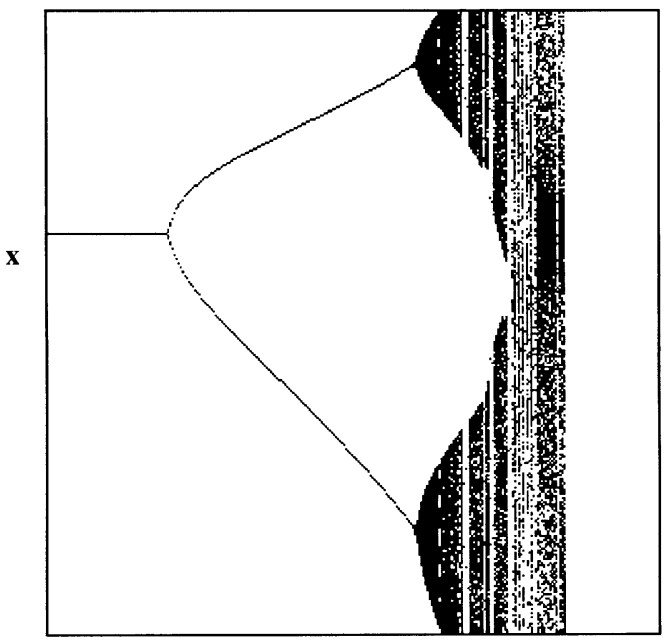

b
Fig. 20. Strange attractor generated by coupling (reproductive) non-oscillating populations $(r=2.8)$
Fig. 21. Bifurcation diagram for $r=2.8$ (reproductive coupling) 


\section{Discussion}

The model discussed here is one special case of coupled logistic maps, for which there is a growing literature (e.g. Froyland, 1983; Paulus et al., 1989; Harikrishnan and Nandakumaran, 1989; Gu et al., 1984; Yuan et al., 1983). The general behavior of a Hopf bifurcation followed by distortion of the torus, quasiperiodic behavior, crisis, and further complicating effects has been frequently observed. Our treatment specifically examines the behavior of the system in three biologically relevant phases, first, when the two populations are independently oscillating in a two point cycle, second when the two populations are independently chaotic, and third, when the two independent populations are independently stable.

The details of bifurcation and chaos generation presented herein are only slightly different from that reported for similar coupled maps previously (e.g. Yuan et al., 1983; Harikrishnan and Nandakumaran). Especially evident is the qualitative similarity of the breakup of the torus following the Hopf bifurcation, discussed fully by Kaneko (1984). The "crisis" behavior discussed by $\mathrm{Gu}$ et al. (1984) is also seen in the present model for a variety of parameter choices. Gu et al., observe two qualitatively distinct crises, a "boundary crisis" and an "interior crisis" (also see Grebogi et al., 1983). The former occurs when a strange attractor collides with an unstable fixed point, clearly resulting in the global instability of the system in general. Such behavior appears to occur at high values of coupling in the present model. An interior crisis is observed (see Fig. 16) for several parameter values and accounts for the sudden loss of one of two alternative attractors. Such behavior might be very interesting biologically in that a population's behavior could easily change quickly from an erratic form to a highly controlled form. For example, with reference to Fig. 16, two coupled populations may be locked into a chaotic pattern that is statistically phase reversed (i.e. Fig. 16b), and with gradual increase in the coupling parameter exhibit no perceptible change in the time series until crisis (Fig. 16d), at which point they move into a highly regular four point cycle.

A variety of ecological situations might mimic such a pattern. For example, two subpopulations of a metapopulation may gradually grow closer together, reaching a crisis point in which their behavior at the metapopulation level dramatically changes from chaotic to regular. Another example might be two consumer resource systems weakly connected through the common consumption of the consumers (e.g. Vandermeer, 1993; 1994). As evolution of generalization procedes (i.e. as the two consumers feed more on each others resources through some selection mechanism), a crisis point will be reached in which phase reversed chaos might quickly and dramatically change to regular behavior (see Fig. 16).

At a general ecological level we report behaviors similar to that reported for coupled consumer resource systems (Vandermeer, 1993; 1994), with either entrainment or phase reversal, followed eventually by chaos, although the exact approach to chaos is substantially different in the maps than in differential equations. But the biological conclusions remain substantially congruent 
whether dealing with differential equations or 1D maps, the populations tend to be locked together at low levels of coupling, becoming chaotic as the coupling intensity increases.

Observations that chaotic populations can be induced to behave nonchaotically through coupling are cited here for the first time, to our knowledge (but see Hastings, 1992). This observation is of interest especially in the growing field of metapopulation modeling. Coupling two maps can be thought of as coupling two subpopulations in a metapopulation together, and the observation that chaos can be abetted through such coupling is of substantial interest. However, such an observation is not a rule, and complicated patterns of chaos, quasiperiodicity, and intermittent chaos are easily generated as a consequence of the coupling phenomenon.

Reproductive coupling promoting phase-reversal has another implication for metapopulations. In contrast to conventional wisdom, increased dispersal under certain conditions could lead to asynchrony among subpopulations, possibly increasing persistence of the metapopulation as a whole. This result has been supported for a metapopulation model composed of 4 subpopulations as well.

Perhaps most surprising in this study is the observation that two populations that are completely stable, that is, characterized by a single fixed point, can be driven into chaotic behavior through the process of coupling. While the observation that coupling can reduce chaos fits in nicely with the general popular idea that metapopulations tend to be stable even when their subpopulations are erratic, the observation that the subpopulations can be stable when isolated, but erratic (chaotic) when linked together is counter intuitive to say the least. We know of no attempt to generate a similar sort of behavior with differential equations, although such a demonstration would be of considerable interest.

\section{References}

Abraham, R. H., Shaw, C. D., 1988. Dynamics - The Geometry of Behavior. Part 4: Bifurcation Behavior. Aerial Press, Santa Cruz, CA. 196 pp

Arrowsmith, D. K., Place, C. M., 1990. An introduction to Dynamical Systems. Cambridge Univ. Press. 423 pp

Bohr, T., Bak, P., Høgh Jensen, M., 1984. Transition to chaos by interaction of resonances in dissipative systems. II. Josephson junctions, charge-density waves, and standard maps. Physical Review A 30: 1970-1981

Buck, J., Buck, E., 1976. Synchronous Fireflies. Scientific American 234: 74-85

Cvitanovic, P., Gunaratne, G. H., Vinson, M. J., 1990. On the mode-locking universality for critical circle maps. Nonlinearity 3: $873-885$

Devaney, R. L., 1986. An introduction to chaotic dynamical systems. W. H. Benjamen

Feigenbaum, M. J., 1979. The universal metric properties of nonlinear transformations. J. Stat. Phys. 19: 25-52

Frøyland, J., 1983. Some symmetric, two-dimensional, dissipative maps. Physica 8D. 423-434

Grebogi, C., Ott, E., Yorke, J. A., 1983. Physica (Utrecht) 7D, 181

Gu, Y., Tung, M., Yuan, J., Feng, D. H., Narducci, L. M., 1984. Crises and hysteresis in coupled logistic maps. Physical Review Letters 52: 701-704 
Harikrishnan, K. P., Nandakumaran, V. M., 1989. An analogue of the logistic map in two dimensions. Physics Letters A 142: 483-489

Hassel, M. P., Comins, H. N., May, R. M.. 1991. Spatial structure and chaos in insect population dynamics. Nature 353: 255-258

Hastings, A., 1992. Complex interactions between dispersal and dynamics: Lessons from coupled logistics, MS

Huffaker, C. B., 1958. Experimental Studies on Predation: Dispersion Factors and Predator-Prey Oscillations. Hilgardia 27: 343-383

Jackson, E. Atlee.. 1991. Perspectives of nonlinear dynamics. Vols I and II. Cambridge Univ. Press, Cambridge, $496 \mathrm{pp}$ and $633 \mathrm{pp}$

Kaneko, K., 1983. Transition from torus to chaos accompanied by frequency lockings with symmetry breaking. Progress of Theoretical Physics, 69: 1427-1442

Lauwerier, H. A., 1986. Two-dimensional iterative maps. In A. V. Holden ed., Chaos, pp 58-95. Princeton University Press, Princeton, N.J.

May, R. M.. 1977. Thresholds and breakpoints in ecosystems with a multiplicity of stable states. Nature 269: 471-477

May, R. M., 1976. Simple mathematical models with very complicated dynamics. Nature 261: 459-467

May, R. M., 1981. Models for Two Interacting Populations. in May. R. M. (ed.) Theoretical Ecology: Principles and Applications. Sinauer Assoc. Inc., Sunderland, Mass

Paulus, M. P., Gass, S. F., Mandell, A. J., 1989. Physica D 40: 135-155

Sachsenmaier, W., Remy, U., Plattner-Schobel, R., 1972. Initiation of Synchronous Mitosis in Physarum polycephalum. Experimental Cell Research 73: 41-48

Torre, V.. 1976. A Theory of Synchronization of Heart Pace-Maker Cells. J. Theor. Biol. 61: $55-71$

van der Pol, B., van der Mark, J., 1927. Frequency demultiplication. Nature 120: 363-364

Vandermeer, J. H.. 1993. Loose coupling of predator prey cycles: entrainment, chaos, and intermittency in the classic MacArthur consumer-resource equations. American Naturalist 141: 687-716

Vandermeer, J. H.. 1994. The qualitative behavior of coupled predator prey oscillations as deduced from simple circle maps. Ecol. Modelling 73: 135-148

Vandermeer, J. H., 1996. Seasonal isochronic forcing of Lotka-Volterra equations. Prog. Theor. Phys. 96: 13-28

Winfree, A. T., 1967. Biological rhythms and the behavior of populations of coupled oscillators. J. Theor. Biol. 16: 15-42

Winful, H. G., Rahman, L., 1990. Synchronized chaos and spatiotemporal chaos in arrays of coupled lasers. Physical Review Letters 65: 1575-1578

Yuan, J., Tung, M., Feng, D. H., Narducci, L., 1983. Instability and irregular behavior of coupled logistic equations. Physical Review A 28: 1662-1666 